\title{
Comparison of circular flexure hinge design equations and the derivation of empirical stiffness formulations
}

\author{
Yuen Kuan Yong and Tien-Fu Lu
}

\begin{abstract}
Flexure hinges are commonly used in many applications which require precise and smooth motions in the nanometer scale. There were various formulations derived using different methods to calculate the stiffness of circular flexure hinges. This article compares these equations with FEA predictions. The limitation of these equations at different $t / R$ ( $R$ is the radius and $t$ is the neck thickness) ratios are revealed. Based on the limitations of these design equations, a guideline to select the most accurate equations for hinge design calculations is presented. In addition to the review and comparisons, general empirical stiffness equations in the $x$ - and $y$-direction were formulated in this study (with errors less than 3\% when compared to FEA simulations) for a wide range of $t / R$ ratios $(0.05 \leq t / R \leq 0.8)$.
\end{abstract}

\section{INTRODUCTION}

Flexure hinges have been widely used in applications such as nanopositioning scanners for scanning probe microscopes [1]-[4], nano-assembly and nano-machining [5][7]. Nanomanipulation has emerged as an important technological advancement that increases the use of flexure hinges. Many micro-motion stages were developed using conventional technologies based on servomotors, ball screws and rigid linkages. However, these conventional technologies encounter problems such as friction, wear, backlash and lubrication, which struggle to achieve high positioning accuracy. Flexure-based mechanisms (also known as compliant mechanisms), which solely move through deformations of flexure hinges, provide smooth motions without encountering these problems. These mechanisms are capable of achieving highly precise positioning with nanometer resolutions.

An accurate analytical or empirical model is needed to obtain a good estimation of workspace or stiffness of a compliant mechanism. A compliant mechanism normally uses a few flexure hinges to provide the desired fine motions in various directions. The accuracy of a compliant stage model relies on the precision of a flexure hinge model. Therefore, compliance (the inverse of stiffness) or stiffness equations of flexure hinges are demanded to be as accurate as possible to reduce the accumulated modeling errors of hinges, subsequently reducing the overall modeling error of compliant mechanisms.

Circular flexure hinges were chosen in this study due to their large applications in compliant micro- and nanopositioning devices [1], [8]-[12]. Circular flexure hinges are

Y.K. Yong is with the School of Electrical Engineering and Computer Science, The University of Newcastle, Callaghan 2308, NSW, Australia. yuenkuan.yongenewcast le.edu. au

T.-F. Lu is with the School of Mechanical Engineering, The University of Adelaide, SA 5005, Australia. tien-fu.lueadelaide.edu.au precise in rotation where their center of rotations do not displace as much as other flexure hinges such as the lefttype [13] and the corner-fillet [14]. There have been many methods adopted to derive satisfactory design equations of flexure hinges, including the integration of linear differential equations of a beam [15]-[17], Castigliano's second theorem [17], inverse conformal mapping [13] and empirical equations formed from finite-element-analysis (FEA) [18], [19]. However, some of these methods provide better accuracies than the others depending on the $t / R$ ratios of circular flexure hinges (see Fig. 1). Three design equations, i.e. the rotational compliance $\Delta \alpha_{z} / M_{z}$, the translational compliance at the $\mathrm{x}$ axis $\Delta x / F_{x}$ and the translational compliance at the $\mathrm{y}$ axis $\Delta y / F_{y}$, are discussed in this paper.

Circular flexure hinges were first introduced in [15]. The full and simplified design equations were formulated. The error of the simplified equation relative to the full equation was within $1 \%$ for hinges with $t / R$ in the range 0.02 to 0.1 , and within $5-12 \%$ for thicker hinges $(0.2 \leq t / R \leq$ 0.6) [13]. However, both the full and simplified rotational compliance equations of $\Delta \alpha_{z} / M_{z}$ show a large difference of $25 \%$ for $t / R=0.6$ when compared with FEA results in [13]. A comparison of experimental results of $M_{z} / \Delta \alpha_{z}$ in [20] with the analytical results in [15] was conducted by the authors. The comparison revealed that the simplified equation shows a difference of up to $10 \%$ and the full equation shows a difference of up to $16 \%$. It was also reported in [20] that the full equation of $\Delta \alpha_{z} / M_{z}$ in [15] had larger errors than the simplified equation when compared with the FEA simulations. Design equations presented in [16] have the same results as the full equations in [15], except signs of $\Delta \alpha_{z} / F_{z}$ and $\Delta y / M_{z}$ were opposite. Therefore, their equations have the same accuracy (neglecting signs).

Rotational compliance equations $\left(\Delta \alpha_{z} / M_{z}\right)$ developed in [13] were categorized into three groups, i.e. thin $(t / R \leq$ $0.07)$, intermediate $(0.07<t / R \leq 0.2)$ and thick $(0.2<$ $t / R \leq 0.6)$, and an equation was derived to calculate compliances for each category respectively. These equations were within $10 \%$ error when comparing with FEA and experimental results. An empirical equation was derived to calculate the rotational compliance of flexure hinges in [18]. This equation is tractable and adequate only for thick hinges with $t / R$ in the range 0.2 to 1.0 as reported in [13]. Closed form compliance equations were derived using the Castigliano's second theorem [21]. The analytical compliance results for hinges, with $t / R$ equal to $0.05,0.1$ and 0.2 , were compared with FEA simulations and the differences were within $10 \%$. There is no comparison of results presented for 


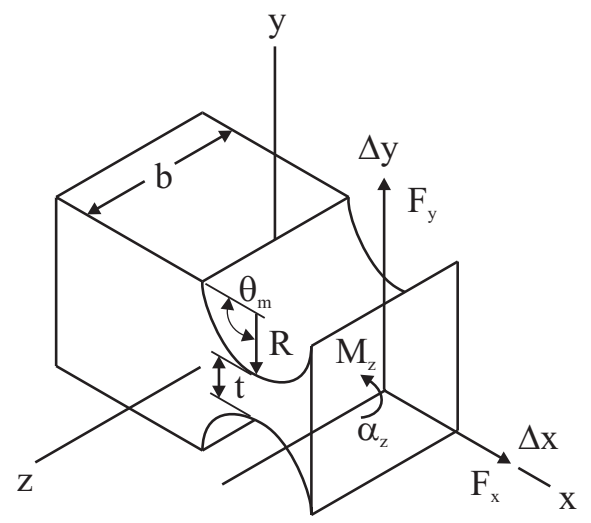

Fig. 1. Flexure hinge. $t$ is the neck thickness. $R$ is the radius of the hinge. $\Delta \alpha_{z}, \Delta x$ and $\Delta y$ are the rotation, $\mathrm{x}$-deformation and $\mathrm{y}$-deformation of the hinge respectively. $M_{z}, F_{x}$ and $F_{y}$ are the moment and forces acting on the corresponding axes. The three design equations discussed in this paper are $\Delta \alpha_{z} / M_{z}, \Delta x / F_{x}$ and $\Delta y / F_{y}$

other $t / R$ ratios.

This article presents a review of the accuracy of circular flexure hinge design equations, at different $t / R$ ratios, based on their differences when compared to FEA predictions. By analyzing the limitations of each design equation, suggestions of the most accurate and appropriate equations to be used at any particular $t / R$ range are provided in this article. In addition to the review and comparisons, general empirical equations were developed based on FEA results to estimate compliance/stiffness in the $\mathrm{x}$ - and $\mathrm{y}$-axis as there is no accurate equation (within 5\%) to predict the corresponding compliance/stiffness of circular flexure hinges for a wide $t / R$ range $(0.05 \leq t / R \leq 0.8)$.

This article is organised as follows. The FEA modeling of circular flexure hinges is presented in Sec. II. Experimental validation of the FEA model is presented in Sec. III. Sec. IV presents the comparison the three design equations $\Delta \alpha_{z} / M_{z}$, $\Delta x / F_{x}$ and $\Delta y / F_{y}$ with FEA simulations. In Sec. V, the derivation of empirical equations of the hinge is presented.

\section{FEA Modeling of Circular Flexure Hinges}

There are only three experimental compliance/stiffness results of flexure hinges found [20] for three $t / R$ cases. Due to the limitated availability of experimental results, FEA simulations were used as a benchmark for comparison for a wide range of $t / R$. The FEA model presented in this paper provides an accuracy of within 3\% when compared with these three experimental results.

ANSYS was used to conduct FEA of flexure hinges. Flexure hinge models were generated using 8-node, twodimensional, plane elements (PLANE82) with two degreeof-freedom on each node, which are translations in the nodal $\mathrm{x}$ and $\mathrm{y}$ directions. This element type is more suitable to model irregular shapes and curved boundaries without much loss of accuracy. The modeled flexure hinge has a thickness of $12.7 \mathrm{~mm}$ (aluminium alloy, 7075-T6) with a Young's modulus $(E)$ of $71.7 \mathrm{GPa}$ and a Poisson ratio $(\nu)$ of 0.33. A mapped meshing technique was used instead

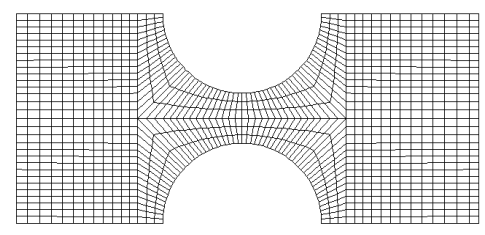

Fig. 2. FEA mapped meshing

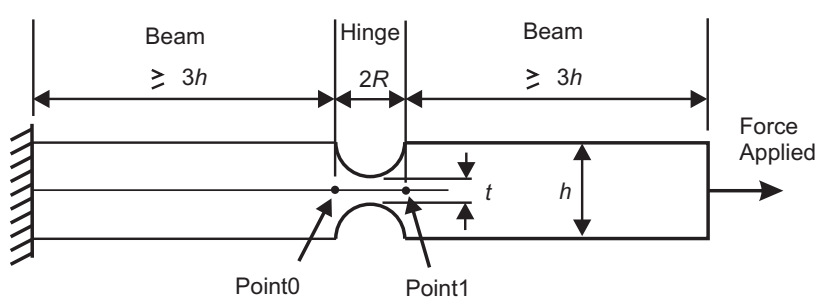

Fig. 3. Flexure hinge geometries of a FEA model

of a "smart" meshing, the latter automatically produces fine meshing at areas that high stress concentrations were most likely to occur. Mapped meshing is advantageous over "smart" meshing because mapped meshing provides better control of the distribution and size of elements in an area (see Fig. 2). It was found that the accuracy of the FEA model was significantly influenced by the way the boundary conditions were assigned on a model. For example, when a point force is applied on a node, it could cause a local stress spike on the node which reduces the accuracy of the FEA results. Therefore, constraints and forces were applied at a distance of at least $3 h$ (see Fig. 3) from a node where its displacements will be read, to reduce influences of the constraints and applied forces on the FEA results. Forces in the $\mathrm{x}$ - and $\mathrm{y}$-direction and a moment along the z-direction were applied. The corresponding nodal deformations at Point 0 and Point 1 were read. Analytical design equations [13], [15]-[18] were derived to calculate compliances at Point 1. However, nodal deformations read from FEA results at Point 1 were the total deformation contributed by the left-hand side beam section as well as the hinge section (see Fig. 3). Therefore, rotations and deformations caused by the beam section (nodal deformations at Point 0 ) were subtracted from total deformations read at Point 1 to obtain pure deformations caused by the hinge.

\section{EXPerimental VAlidation of the FEA Model}

Three flexure hinges from each of the categories of thin $(t / R \leq 0.07)$, intermediate $(0.07<t / R \leq 0.2)$ and thick $(0.2<t / R \leq 0.8)$ circular hinges were selected for experimental study to verify the accuracy of the FEA model. Unfortunately, a consistent result of the $\Delta x$-compliance $\left(\Delta x / F_{x}\right)$ could not be obtained due to the limitation of sensor resolution. However, the order of accuracy of the $\Delta x$ compliance is approximately the same as the $\Delta \alpha_{z^{-}}$and $\Delta y$ compliances. This assumption was made because the FEA modelling concept of determining the $\Delta x$-compliance was the same as that of the $\Delta \alpha_{z^{-}}$and $\Delta y$-compliances. 
The experimental setup of measuring the $\Delta \alpha_{z^{-}}$and $\Delta y$ compliance is shown in Figure 4. The experiment was setup on an anti-vibration table. For the measurement of $\Delta \alpha_{z}$-compliance, two eddy-current sensors (3316 and 3317) were used to detect deformations of the hinge. For the measurement of $\Delta y$-compliance, a fibre-optic sensor was used instead of the two eddy-current sensors. This is because the fibre-optic sensor has a smaller resolution which is more suited for measuring the small $\Delta y$-deformation of flexure hinges. Forces were applied on the flexure hinges by hanging weights at a predetermined position.

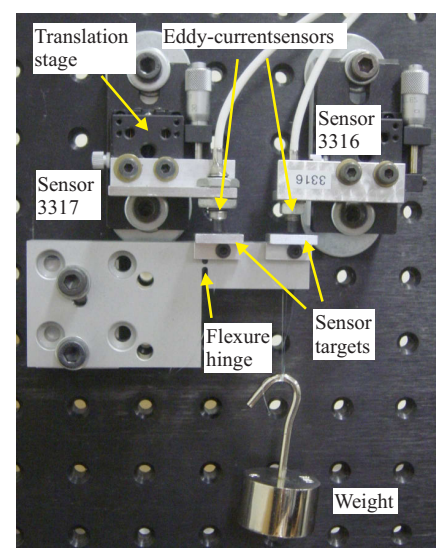

(a)

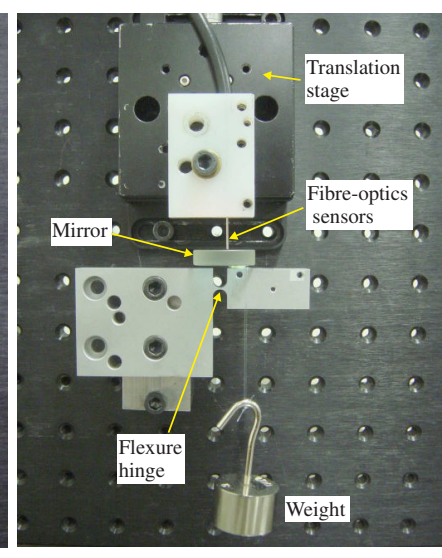

(b)
Fig. 4. Experimental setup of the (a) $\Delta \alpha_{z}$-compliance and (b) $\Delta y$ compliance measurement.

The $\Delta \alpha_{z}$-deformation of a flexure hinge can be calculated easily from deformations detected by the two sensors. Figure 5(a) shows the schematic of the experimental setup and dimensions.

The $\Delta \alpha$ - compliance $\left(\Delta \alpha_{z} / M_{z}\right)$ is calculated as,

$$
\frac{\Delta \alpha_{z}}{M_{z}}=\frac{\Delta \alpha_{z}}{F_{1}\left(L_{1}+R\right)}
$$

where $\Delta \alpha_{z}=\arctan \left(\frac{d_{1}-d_{2}}{L_{1}-L_{2}}\right) \cdot d_{1}$ and $d_{2}$ are the deformation measured by the sensors.

The overall y-axis deformation measured by the fibre-optic sensor consists of a deformation component caused by a force, $F_{y}=F_{2}$ and a deformation component caused by a moment, $M_{z 2}=F_{2} L_{2}$ (see Figure 5(b)). In order to calculate the $\Delta y$-deformation and compliance which are only caused by $F_{y}$, the deformation caused by $M_{z 2}$ is required to be subtracted from the measured results.

The $\Delta y$-deformation caused by $M_{z 2}$ is $\Delta y_{M_{z 2}}=$ $\left(\Delta \alpha_{z} / M_{z}\right) \cdot M_{z 2} \cdot R$, where $\Delta \alpha_{z} / M_{z}$ is determined from Eq. 1. Thus, the $\Delta y$-deformation caused by $F_{y}$ is,

$$
\Delta y=\Delta y_{\text {fibre-optics }}-\Delta y_{M_{z 2}}
$$

The $\Delta y$-compliance $\left(\Delta y / F_{y}\right)$ of the hinge is then calculated by dividing Equation 2 with $F_{y}$.
TABLE I

COMPARISON BETWEEN FEA AND EXPERIMENTAL RESULTS

\begin{tabular}{ccccccccc}
\hline \multicolumn{3}{c}{ Flexure hinge $(\mathrm{mm})$} & \multicolumn{3}{c}{$\Delta \alpha_{z} / M_{z}(\mathrm{rad} / \mathrm{Nm})$} & \multicolumn{3}{c}{$\Delta y / F_{y}(\mu \mathrm{m} / \mathrm{N})$} \\
$t$ & $R$ & $t / R$ & FEA & Exp. & $\%$ diff. & FEA & Exp. & $\%$ diff. \\
\hline 0.5 & 3 & 0.167 & 0.157 & 0.159 & -1.0 & 1.518 & 1.477 & 2.8 \\
0.7 & 1.87 & 0.374 & 0.056 & 0.055 & 1.3 & 0.227 & 0.218 & 4.3 \\
0.84 & 1.1 & 0.764 & 0.029 & 0.028 & 2.6 & 0.047 & 0.044 & 6.2 \\
\hline
\end{tabular}

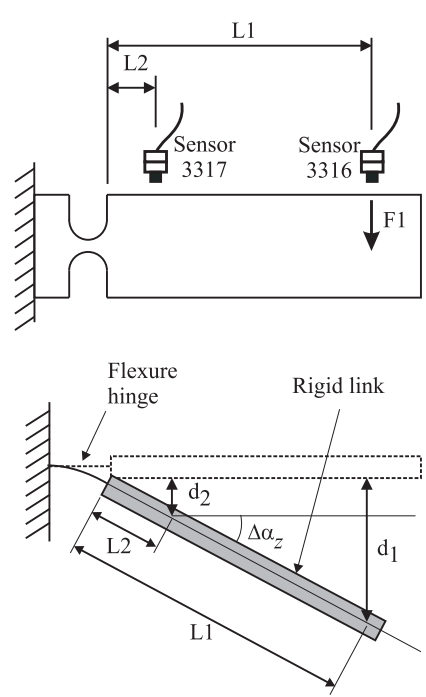

(a)

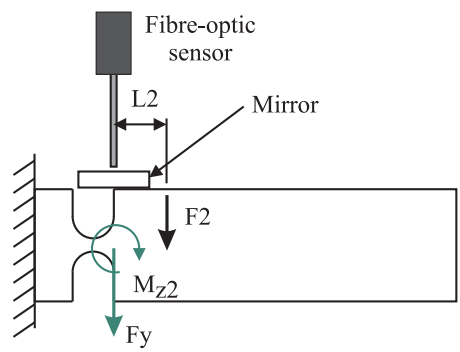

(b)

Fig. 5. Schematics of the (a) $\Delta \alpha_{z}$-compliance and (b) $\Delta y$-compliance measurement of flexure hinges.

Table I shows the comparison of compliances between FEA and experimental results. The FEA determined $\Delta \alpha_{z^{-}}$ compliances were within $3 \%$ of the experimental results while the $\Delta y$-compliances were within $6 \%$ of the experimental compliances. These results indicated that the developed FEA model was defined with appropriate boundary conditions and mesh sizes, and the model is capable of providing good accuracies of compliance results for flexure hinges with different $t / R$ ratios. The comparison of results also confirmed that the FEA model can be used as a benchmark for comparisons with other analytical results without much loss of accuracy.

\section{COMPARison OF COMPliance Results With FEA}

\section{A. Rotational compliance equations, $\Delta \alpha_{z} / M_{z}$}

Compliances, $\Delta \alpha_{z} / M_{z}$ of different circular flexure hinges (with various $t$ and $R$ values), where $t / R$ in a range 0.05 to 


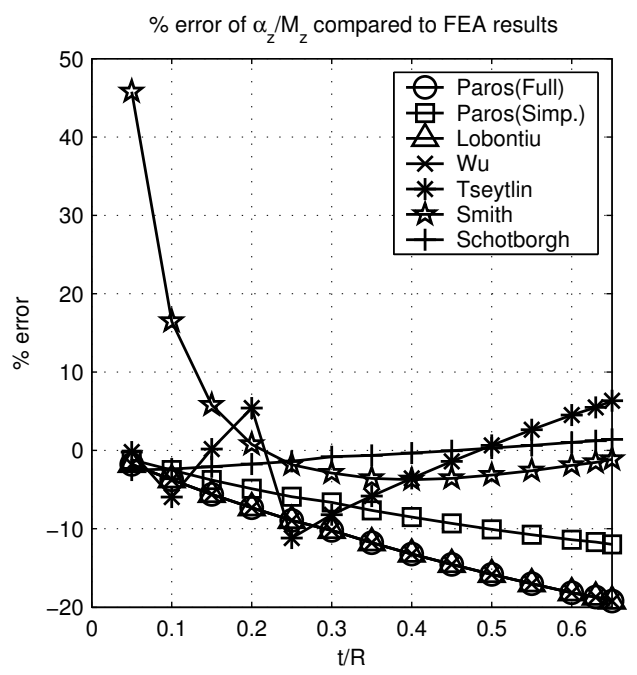

Fig. 6. Percentage differences of $\Delta \alpha_{z} / M_{z}$ compared to FEA results.

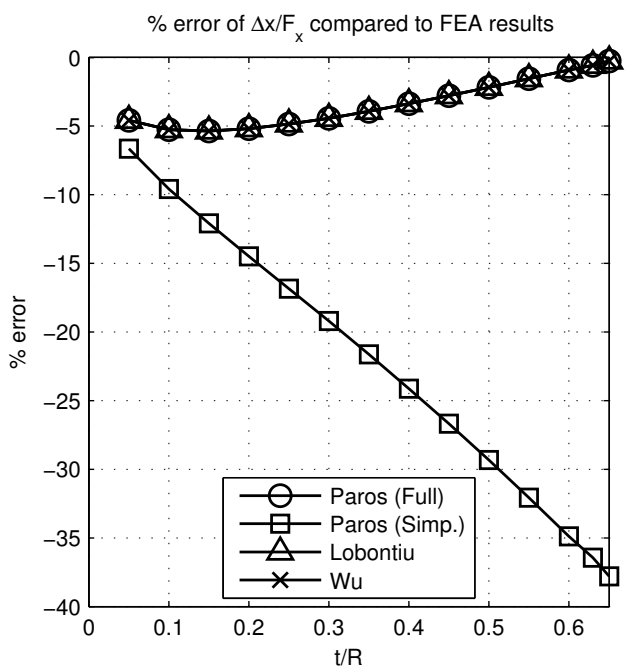

Fig. 7. Percentage differences of $\Delta x / F_{x}$ compared to FEA results

0.65 , were calculated using design equations of a) Paros and Weisbord [15] (full), b) Paros and Weisbord [15] (simplified), c) Lobontiu [17], d) Wu and Zhou [16], e) Tseytlin [13], f) Smith et al. [18] (empirical) and g) Schotborgh et al. [19] (empirical). A concise list of these design equations can be found in [22]. Their results were compared with the FEA simulations obtained in Sec. II. The differences are shown in Fig. 6. It is noted that

- equations of Paros and Weisbord (full), Lobontiu and $\mathrm{Wu}$ and Zhou were derived using a similar method (that is the integration of the linear differential equation of a beam); thus they have the same accuracy when compared with FEA simulations. The accuracies of their results decrease over the $t / R$ range. Differences are less than $5 \%$ when $0.05 \leq t / R<0.15$. Differences increase to more than $5 \%$ but less than $10 \%$ when $0.15 \leq t / R<$ 0.3 . When $0.3 \leq t / R \leq 0.65$, differences increase from $10 \%$ to $19.2 \%$
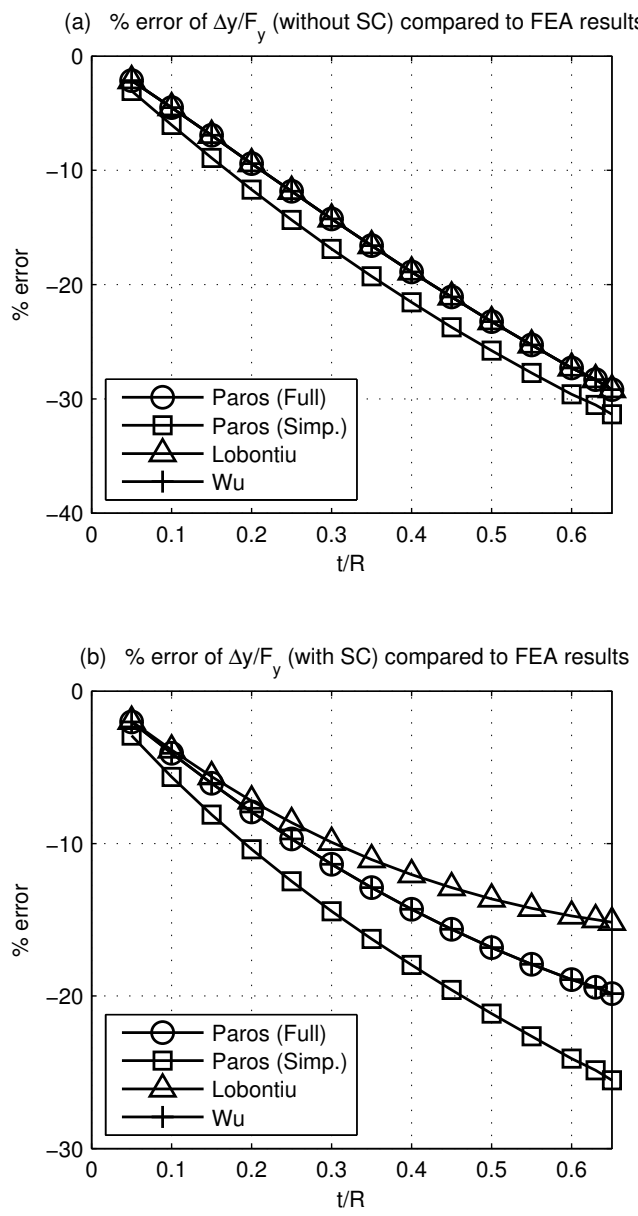

Fig. 8. Percentage differences of $\Delta y / F_{y}$ compared to FEA results. (a) without shear compliance. (b) with shear compliance

- Paros and Weisbord's (simplified) results are accurate within $5 \%$ difference for $0.05 \leq t / R \leq 0.2$. Differences increase up to $10 \%$ when $t / R=0.5$ and $12 \%$ when $t / R=0.65$

- Tseytlin's results are accurate (within $6 \%$ difference) for $t / R \leq 0.23$ and $0.35 \leq t / R<0.65$. Differences increase to more than $6 \%$ when $0.23<t / R<0.35$. When $t / R=0.25$, the difference is $11.2 \%$. Generally, the results are within $10 \%$ difference approximately for the entire $t / R$ range. Interestingly, Tseytlin's analytical results differed from his experimental and FEA results by about $10 \%$ [13]. This suggests the accuracy of FEA results in this article match the accuracy of Tseytlin's analytical and FEA results.

- Smith et al.'s (empirical) rotational compliance equation is only accurate (within $4 \%$ difference) for $0.20 \leq$ $t / R \leq 0.65$. This observation is similar to that of Tseytlin where he claimed that Smith et al.'s empirical equation is accurate only for thick flexure hinges with $t / R$ in the range 0.2 to 1.0

- Schotborgh et al.'s results are the most accurate compared to the rest. Differences in their results are less than $2.5 \%$ for the whole $0.05 \leq t / R \leq 0.65$ range 
B. Compliance equations in the $x$ - and $y$-direction, $\Delta x / F_{x}$ and $\Delta y / F_{y}$

Differences of $\Delta x / F_{x}$ and $\Delta y / F_{y}$, calculated using design equations of a) Paros and Weisbord [15] (full), b) Paros and Weisbord [15] (simplified), c) Lobontiu [17] and d) Wu and Zhou [16] were plotted in Figs. 7 and 8 respectively. For $\Delta x / F_{x}$,

- Paros and Weisbord (full), Lobontiu, and Wu and Zhou have the same results. Their results are within $6 \%$ difference compared to FEA results

- results of Paros and Weisbord (simplified) are not as accurate as that of Paros and Weisbord (full), Lobontiu, and $\mathrm{Wu}$ and Zhou. Their results have a minimum difference of $6.6 \%$ and a maximum difference of $38 \%$

For $\Delta y / F_{y}$ (without shear compliance, SC),

- results of Paros and Weisbord (full), Lobontiu, and Wu and Zhou, without considering shear compliance (SC), are the same. Their differences are within $10 \%$ for $0.05 \leq t / R \leq 0.2$. differences increase up to $30 \%$ when $t / R=0.65$

- Paros and Weisbord's (simplified) results are within $10 \%$ when $0.05 \leq t / R \leq 0.17$. Differences increase to $31 \%$ when $t / R=0.65$

For $\Delta y / F_{y}$ (with shear compliance, $\mathrm{SC}$ ),

- Lobontiu's results are the most accurate compared to the others. The results are within 5\% difference for $0.05 \leq$ $t / R \leq 0.1$. The differences increase to within $10 \%$ for $0.1<t / R \leq 0.3$ and to within $15 \%$ for $0.3<t / R \leq$ 0.65

- results of Paros and Weisbord's (full) and Wu and Zhou are the same. Their results are within 5\% difference for $0.05 \leq t / R \leq 0.1$. The differences increase to within $10 \%$ for $0.1<t / R \leq 0.25$ and to within $20 \%$ for $0.25<t / R \leq 0.65$

- Paros and Weisbord's (simplified) results are within 5\% difference for $0.05 \leq t / R<0.1$. The differences increase to within $10 \%$ for $0.1 \leq t / R \leq 0.17$. The maximum difference is $25.5 \%$ when $t / R=0.65$

Generally, all compliance equations, $\Delta y / F_{y}$ are more accurate when shear compliances are considered. Schotborgh's empirical stiffness/compliance equations in the $\mathrm{x}$ - and $\mathrm{y}$ direction were not compared in this article because his equations were derived using hinge models with different height ( $h$, see Fig. 3). Furthermore, his empirical equations consider the deformation effect outside the hinge geometry (as discussed via a personal communication with Schotborgh). Other research groups [15]-[17] did not consider this effect in their equations. The aspect of this deformation effect on the hinge compliances is beyond the scope of this paper. The significance of this effect are currently being investigated and will be revealed in the future.

\section{EMPIRICAL STIFFNESS EQUations IN THE X-AND Y-DIRECTION}

From previous section, it was noted that there is no accurate design equations (within 5\% difference) at this stage
TABLE II

COEFFICIENTS OF POLYNOMIAL FUNCTIONS FOR $K_{x}$ AND $K_{y}$

\begin{tabular}{ccc}
\hline Coefficients & $K_{x}$, 5th order & $K_{y}$, 6th order \\
\hline$c_{0}$ & 0.036343 & $1.92 \times 10^{-5}$ \\
$c_{1}$ & 0.98683 & -0.00083463 \\
$c_{2}$ & -1.5469 & 0.021734 \\
$c_{3}$ & 3.1152 & 0.064783 \\
$c_{4}$ & -3.0831 & -0.088075 \\
$c_{5}$ & 1.2031 & 0.062278 \\
$c_{6}$ & - & -0.018781 \\
\hline
\end{tabular}

to estimate compliances/stiffness in the y-direction for $t / R>$ 0.15 and in the $\mathrm{x}$-direction for $0.1<t / R<0.25$. Therefore, general empirical equations (in stiffness form, named $K_{x}$ and $K_{y}$ ) were formed based on FEA results to estimate stiffness in both the $\mathrm{x}$ - and $\mathrm{y}$-direction for a wide range of $t / R$ ratios $(0.05 \leq t / R \leq 0.8)$. FEA models with various $t / R$ ratios, which was set from 0.05 to 0.8 with an increment of 0.01 , were generated in ANSYS. Unit forces, $F_{x}$ and $F_{y}$ were applied on each model and the corresponding deformations, $\Delta x$ and $\Delta y$ were read. Polynomial functions with $3^{r d}, 4^{t h}$, $5^{\text {th }}$ and $6^{\text {th }}$ order were fitted through the data points to obtain empirical stiffness equations. The results of these four empirical equations were compared with FEA results and the differences were plotted in Figs. 9 and 10. $5^{\text {th }}$ and $6^{\text {th }}$ order polynomial functions were used for $K_{x}$ and $K_{y}$ respectively in order to keep the differences as small as possible. By using the two polynomial functions, maximum differences occurred when $t / R=0.05$, which are $1.1 \%$ and $2.7 \%$ for $K_{x}$ and $K_{y}$ respectively. Both empirical equations are shown in Eq. 3. Table II exhibits coefficients of these equations.

$$
K_{x}=E b\left[\sum_{k=0}^{n} c_{k}\left(\frac{t}{R}\right)^{k}\right], K_{y}=E b\left[\sum_{k=0}^{n} c_{k}\left(\frac{t}{R}\right)^{k}\right]
$$

where $c_{k}$ are coefficients of polynomial functions, $\mathrm{n}$ is the order of a polynomial function.

Finally, Table III summarizes the suggested compliance/stiffness equations to be used for any particular $t / R$ range, and their minimum, maximum and average differences.

\section{CONClusions}

This article reviews the accuracy of previously derived design equations of circular flexure hinges. Based on the review, a guideline on how to select the most suitable compliance/stiffness design equation for a particular $t / R$ value is presented. FEA simulations were used as a benchmark to compare various design equations of flexure hinges for a wide $t / R$ range. In addition to the review and comparisons, general empirical stiffness equations in the $\mathrm{x}$ - and $\mathrm{y}$-direction were formulated in this study for $0.05 \leq t / R \leq 0.8$. The differences of these empirical equations were found to be less than 3\% when compared to FEA simulations.

\section{ACKNOWLEDGMENTS}

The authors would like to greatly acknowledge the support of the Adelaide Robotics Research Group at the University 
TABLE III

SUGGESTED COMPLIANCE/STIFFNESS EQUATIONS FOR A PARTICULAR $t / R$ RANGE

\begin{tabular}{|c|c|c|c|c|c|c|c|c|c|c|c|c|}
\hline \multirow{2}{*}{$\begin{array}{l}\text { Research } \\
\text { Group }\end{array}$} & \multirow{2}{*}{$\begin{array}{l}\Delta \alpha_{z} / M_{z} \\
t / R \text { range }\end{array}$} & \multicolumn{3}{|c|}{$\%$ difference } & \multirow{2}{*}{$\begin{array}{c}\Delta y / F_{y}(\text { with } \mathrm{SC}) \\
t / R \text { range }\end{array}$} & \multicolumn{3}{|c|}{$\%$ difference } & \multirow{2}{*}{$\begin{array}{c}\Delta x / F_{x} \\
t / R \text { range }\end{array}$} & \multicolumn{3}{|c|}{$\%$ difference } \\
\hline & & $\min$. & $\max$. & ave. & & $\min$. & $\max$. & ave. & & $\min$. & $\max$. & ave. \\
\hline Paros (full) [15] & $0.05 \leq t / R<0.1$ & 1.8 & 5.0 & 3.5 & $0.05 \leq t / R \leq 0.1$ & 2 & 4 & 3.1 & $0.25 \leq t / R \leq 0.65$ & 0.3 & 4.9 & 2.4 \\
\hline Paros (simpl.) [15] & $0.05 \leq t / R \leq 0.2$ & 1.2 & 4.9 & 3.1 & $0.05 \leq t / R \leq 0.1$ & 3 & 5.6 & 4.3 & \multicolumn{4}{|c|}{ Not recommended } \\
\hline Lobontiu [23] & $0.05 \leq t / R<0.1$ & 1.8 & 5.0 & 3.5 & $0.05 \leq t / R \leq 0.1$ & 2 & 3.9 & 2.9 & $0.25 \leq t / R \leq 0.65$ & 0.3 & 4.9 & 2.4 \\
\hline Wu and Zhou [16] & $0.05 \leq t / R<0.1$ & 1.8 & 5.0 & 3.5 & $0.05 \leq t / R \leq 0.1$ & 2 & 4 & 3.1 & $0.25 \leq t / R \leq 0.65$ & 0.3 & 4.9 & 2.4 \\
\hline Tseytlin [13] & $0.4 \leq t / R \leq 0.6$ & 0.7 & 4.5 & 2.5 & NA & NA & NA & NA & NA & NA & NA & NA \\
\hline Smith [18] & $0.2 \leq t / R \leq 0.65$ & 0.8 & 3.7 & 2.4 & NA & NA & NA & NA & NA & NA & NA & NA \\
\hline Schotborgh [19] & $0.05 \leq t / R \leq 0.65$ & 0.03 & 2.5 & 1.2 & NA & NA & NA & NA & NA & NA & NA & NA \\
\hline This article & NA & NA & NA & NA & $0.05 \leq t / R \leq 0.8$ & 0 & 2.7 & 0.07 & $0.05 \leq t / R \leq 0.8$ & 0 & 1.1 & 0.08 \\
\hline
\end{tabular}

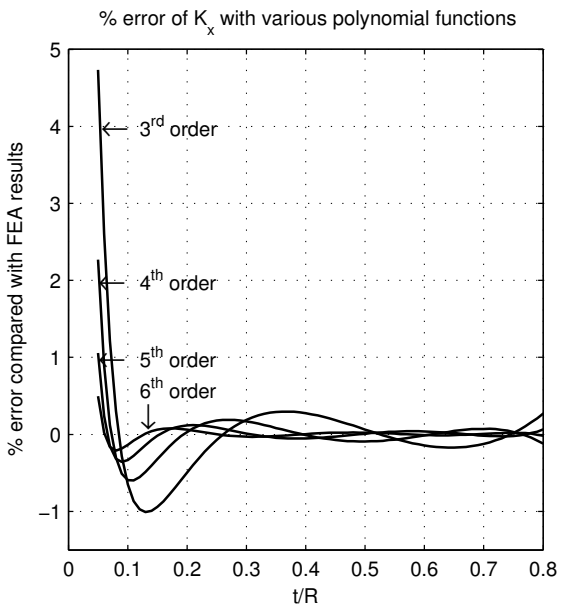

Fig. 9. Accuracy of the empirical equations, $K_{x}$ represented by the $3 \mathrm{rd}$, 4th, 5th and 6th order polynomial functions.

of Adelaide and the use of its facilities. Also, a special thanks to Dr. Andrei Kotousov and Dr. Carl Howard for their great advices on FEA modelling and their valuable time.

\section{REFERENCES}

[1] Y. K. Yong, S. Aphale, and S. O. R. Moheimani, "Design, identification and control of a flexure-based XY stage for fast nanoscale positioning," IEEE Trans. Nanotechnology, vol. 8, no. 1, pp. 46-54, 2009.

[2] T. Ando, N. Kodera, T. Uchihashi, A. Miyagi, R. Nakakita, H. Yamashita, and K. Matada, "High-speed atomic force microscope for capturing dynamic behavior of protein molecules at work," e-Journal of Surface Science and Nanotechnology, vol. 3, pp. 384-392, 2005.

[3] G. Schitter, K. J. Åstrom, B. DeMartini, P. J. Thurner, K. L. Turner, and P. K. Hansma, "Design and modeling of a high-speed AFM-scanner," IEEE Trans. Contr. Syst. Tech., vol. 15, no. 5, pp. 906-915, 2007.

[4] S. Salapaka, A. Sebastian, J. P. Cleveland, and M. V. Salapaka, "High bandwidth nano-positioner: A robust control approach," Rev. Sci. Instrum., vol. 73, no. 9, pp. 3232-3241, 2002.

[5] B. Bhushan, Ed., Springer Handbook of Nanotechnology, 2nd ed. Springer-Verlag, 2004.

[6] S. Devasia, E. Eleftheriou, and S. O. R. Moheimani, "A survey of control issues in nanopositioning," IEEE Trans. Contr. Syst. Tech., vol. 15 , pp. 802-823, 2007.

[7] G. M. Whitesides and J. C. Love, "The art of building small," Scientific American, vol. 285, no. 3, pp. 32-41, Sept. 2001.

[8] Y. K. Yong and T.-F. Lu, "The effect of the accuracies of flexure hinge equations on the output compliances of planar micro-motion stages," Mechanism and Machine Theory, vol. 43, pp. 347-363, 2008.

[9] F. Scire and E. Teague, "Piezodriven 50- $\mu \mathrm{m}$ range stage with subnanometer resolution," Rev. Sci. Instrum., vol. 49, no. 12, pp. 17351740, 1978.

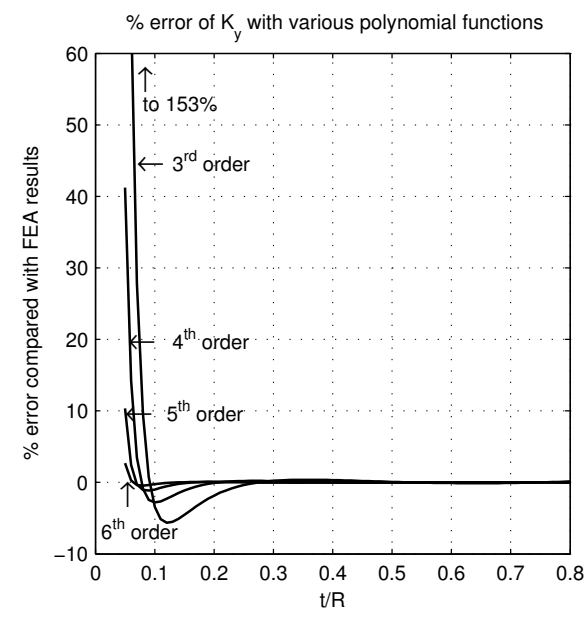

Fig. 10. Accuracy of the empirical equations, $K_{y}$ represented by the $3 \mathrm{rd}$, 4 th, 5th and 6th order polynomial functions.

[10] P. Gao, S. Swei, and Z. Yuan, "A new piezodriven precision micropositioning stage utilizing flexure hinges," Nanotechnology, vol. 10, pp. 394-398, 1999.

[11] I. Her and J. Chang, "A linear scheme for the displacement analysis of micropositioning stages with flexure hinges," J. Mech. Des., vol. 116, pp. 770-776, 1994.

[12] M. Jouaneh and P. Ge, "Modelling and control of a micro-positioning tower," Mechatronics, vol. 7, no. 5, pp. 465-478, 1997.

[13] Y. Tseytlin, "Notch flexure hinges: An effective theory," Rev. Sci. Instrum., vol. 73, no. 9, pp. 3363-3368, 2002.

[14] N. Lobontiu, J. Paine, E. Garcia, and M. Goldfarb, "Corner-filleted flexure hinges," J. Mech. Des., vol. 123, pp. 346-352, 2001.

[15] J. Paros and L. Weisbord, "How to design flexure hinge," Mach. Des., vol. 37, pp. 151-156, 1965.

[16] Y. Wu and Z. Zhou, "Design calculations for flexure hinges," Rev. Sci. Instrum., vol. 73, no. 9, pp. 3101-3106, 2002.

[17] N. Lobontiu, Compliant Mechanisms: Design of flexure hinges. CRC Press, 2003.

[18] S. Smith, D. Chetwynd, and D. Bowen, "Design and assessment of monolithic high precision translation mechanisms," J. Phys. E, vol. 20, pp. 977-983, 1987.

[19] W. Schotborgh, F. Kokkeler, H. Trager, and F. van Houten, "Dimensionless design graphs for flexure elements and a comparison between three flexure elements," Precis. Eng., vol. 29, pp. 41-47, 2005.

[20] S. Smith, V. Badami, J. Dale, and Y. Xu, "Elliptical flexure hinges," Rev. Sci. Instrum., vol. 68, no. 3, pp. 1474-1483, 1997.

[21] N. Lobontiu, J. Paine, E. Garcia, and M. Goldfarb, "Design of symmetric conic-section flexure hinges based on closed-form compliance equations," Mech. Mach. Theory, vol. 37, pp. 477-498, 2002.

[22] Y. K. Yong, T.-F. Lu, and D. C. Handley, "Review of circular flexure hinge design equations and derivation of empirical formulations," Precision Engineering, vol. 32, no. 2, pp. 63-70, 2008.

[23] N. Lobontiu, Compliant Mechanisms: Design of flexure hinges. CRC Press, 2003. 